\title{
Persian Version of Functional Assessment of Cancer Therapy- Breast (FACT-B) Scale: Confirmatory Factor Analysis and Psychometric Properties
}

\author{
Mozhgan Patoo ${ }^{1 *}$, Abbas Ali Allahyari ${ }^{1}$ Ali Reza Moradi², Mehrdad Payandeh ${ }^{3}$
}

\begin{abstract}
Background: The Functional Assessment of Cancer Therapy - Breast (FACT-B) scale is widely used to measure health-related quality of life in cancer patients. The aim of the present study is to validate the FACT-B in a sample of Iranian women with breast cancer. Materials and Methods: The sample consisted of 300 women selected through non-random convenient sampling procedure from oncology hospitals and clinics in Kermanshah and Shiraz cities. They were asked to fill in the Persian versions of the FACT-B scale, Hospital Anxiety and Depression Scale, the European Organization for Research and Treatment of Cancer quality of life EORTC QLQ30. Confirmatory factorial analysis of the methods, concurrent validity and discriminant, and Cronbach's alpha for internal consistency were applied. Results: Internal consistency using Cronbach's alpha was 0.63 to 0.93 for the subscales and 0.92 for the total scale. Significant correlations between FACT- B and other measures indicate that this scale had concurrent and discriminant validity. The values of fit indices were satisfactory. Conclusions: The Persian version of the FACT-B scale is valid and reliable and, therefore, the scale can be used in research and clinical settings to assess health-related quality of life in Iranian patients with breast cancer.
\end{abstract}

Keywords: HRQoL - FACT-B scale - breast cancer - psychometric properties - Iran

Asian Pac J Cancer Prev, 16 (9), 3799-3803

\section{Introduction}

Health- related quality of life (HRQoL) first used by Cella and Tulsky (1993) measures the discrepancy between the patients' actual health state and the ideal functioning, satisfaction and well-being state. HRQoL refers to the extent to which one's usual or expected physical, emotional and social well-being affected by a medical condition and/or its treatment (Cella and Tulsky, 1993). HRQoL is a multidimensional concept and a difficult concept to define because of composed of broad domains that generally include physical, emotional, functional and social well-being. It has become increasingly common for HRQoL to be included as an important endpoint in largescale clinical trials investigating the effects of anticancer treatment. (Victorson, 2008). Studies of HRQoL can indicate the directions needed for more efficient treatment of cancer patients and measurement of HRQoL provides information that is useful for understanding how disease and treatments affect the daily life of patients. Indeed health is not simply a measure of either absence or Health is not simply a measure of either absence or presence of disease but also includes social and physical functioning as well (Manandhar et al., 2014). Therefore HRQoL is a significant area of concern in the treatment of patients with cancer (Montazeri, 2008).

Among HRQoL studies in cancer patients, breast cancer has received most attention for several reasons (Munoz, 2010). First, the number of women with breast cancer is increasing. Secondly, early detection and treatment of breast cancer have improved and survivors now live longer. Thirdly, surgery for breast cancer significantly affects a woman's self-image and HRQoL studies can measure this (Munoz, 2010). Also research findings have indicated that the diagnosis of breast cancer is associated with heightened levels of negative emotions and psychological distress, especially symptoms of anxiety and depression (Ramadas et al., 2015). Therefore breast cancer requires special care and consideration.

There are many tests or instruments that have been used in the context of cancer patients to measure the degree of impact on the HRQoL of the patient following the diagnosis or treatment of cancer. HRQOL assessments need to be rapid, facile, and still be aggregated in a clinically meaningful and psychometrically acceptable manner to capture the most relevant patient issues (Yanez et al., 2013). One of the most frequently used tests is the Functional Assessment of Cancer Therapy - Breast 
(FACT-B) Scale (Brady et al., 1997; Cella et al., 1993). It has been translated into over 50 languages, such as Korean, Malayalam, Japanese and Chinese etc. The aim of the present study is to test the reliability and validity of the FACT-B scale in a sample of Iranian cancer breast patients.

\section{Materials and Methods}

\section{Participants}

Three hundred women were recruited from the oncology clinics and hospitals in Kermanshah and Shiraz cities, Iran. This includes breast cancer diagnoses, outpatients or inpatients with a pathologic diagnosis of cancer with any type or stage. The sample completed a self-administered questionnaire, Functional Assessment of Cancer Therapy - Breast (FACT-B) Scale EORTC QLQ30 and Hospital Anxiety and Depression Scale. A summary of the medical and socio-demographic characteristics can be found in Table 1 .

\section{Instruments}

The FACT-B (V4.0) consists of two parts: the general subscale on cancer (FACT-G) and additional concerns on breast cancer-specific subscale (BCS). The FACT-G includes physical well-being (PWB, seven items), social/ family well-being (SFWB, seven items), emotional wellbeing (EWB, six items), and functional well-being (FWB, seven items) and the BCS has nine items. Each item is rated on a 5-point Likert scale. The FACT-B total score is the sum of scores of all five subscales, and can range from 0 to 144. A higher score indicates a better HRQoL of the patient (Cella et al, 1993; 1997).

\section{Persian version of the FACT-B}

The 'forward-backward' procedure was applied to translate the FACT-B from English into Persian. After obtaining copyright permission from the original author, the scale by was translated from English to Persian by an expert in English and a PhD student in psychology, and next revised by a professor of psychology. Another language expert translated back it into English to be compared with the original form. The final version to obtain the content validity was provided to another professor of psychology evaluate the scale from content and clarity partial that led to reforms (improvements) in some items.

\section{EORTC QLQ30}

The EORTC QLQ-C30 (version 3) (Aaronson et al. 1993; Fayers et al. 2001) is a 30-item questionnaire composed of nine sub-scales and six single items: five functioning sub-scales (physical, role, mental, cognitive and social functioning), three symptom sub-scales (fatigue, nausea/vomiting and pain), one global Qol and six single items (dyspnoea, sleep disturbance, appetite loss, constipation, diarrhoea and the financial impact of cancer). Ratings for each item are scored from 1 (not at all) to 4 (very much).The QLQ-C30 was scored according to algorithms recommended by the EORTC. Higher scores represent better function or higher levels of symptoms. This scale was translated and validated to Persian by
Montazeri et al. Crohnbach's alpha coefficient for multiitem scales (to test reliability) ranged from 0.48 to 0.95 at baseline and from 0.52 to 0.98 at follow-up administration of the questionnaire. The Persian version of the EORTC QLQ-C30 is a reliable and valid measure of quality of life in cancer patients and can be used in clinical trials and studies of outcome research in oncology (Montazeri, 1999).

\section{The Hospital Anxiety and Depression Scale (HADS)}

The HADS contains 14 items and consists of two subscales: anxiety ( 7 items) and depression ( 7 items). Each item is rated on a four-point scale, giving maximum scores of 21 for anxiety and depression. The scores of 11 or more on either subscale are considered to be a significant 'case' of psychological morbidity, while the scores between 8-10 represent 'borderline' and 0-7 are 'normal'. The HADS has been previously validated for the Iranian population .This preliminary validation study of the Persian version of the HADS proves that it is an acceptable, reliable and valid measure of psychological distress among cancer patients (Montazeri, 2003). In this study HADS is used for examine construct validity of Mini-MAC scale.

\section{Statistical analysis}

The data were analyzed using SPSS 15.0. Internal reliability was examined by using Cronbach's alpha coefficient. To assess construct validity the inter scale correlations were used. Correlations of the FACT-B factors scores with EORTC QLQ-C30 and Hospital Anxiety and Depression Scale scores were also calculated for discriminant and concurrent validity. A confirmatory factor analysis was carried out on BACT-B scale items using the LISREL 8.50 procedure.

\section{Results}

\section{Descriptive statistics}

Socio-demographic and clinical characteristics of the population are presented in Table 1 . In the sample, the mean age is 47.27 years (The range age $16-85$ ). The marital status of patients: single, 25 patients, 260 patients married, 11 divorced, and 12 widowed. Employment status: 79 patients employed, 214 patients unemployed, and 27 patients uncertain.

Table 1. Socio-demographic and Medical Data of the Patients $(\mathrm{N}=300)$

\begin{tabular}{llll}
\hline & & $\mathrm{N}$ & $\%$ \\
\hline Mean Age & & 43.34 & \\
Rang Age & & $23-72$ & \\
Marital status & Married & 247 & 84 \\
& Never-married & 16 & 8 \\
& Divorced & 10 & 3.5 \\
& Widowed & 9 & 4 \\
Occupation & Employed & 59 & 26 \\
& Unemployed/ Retired & 183 & 65 \\
& Unknown & 59 & 9 \\
Treatment & Surgery & 76 & 25.2 \\
& Chemotherapy & 73 & 24.3 \\
& Radiotherapy & 62 & 20.6 \\
& Other/unknown & 90 & 29.9 \\
\hline
\end{tabular}




\section{Reliability}

Internal consistency of the subscales Of Mini-MAC is done by calculating Cronbach's alpha coefficients. Table 2 shows the Cronbach's a coefficient values of the FACT-B. Cronbach's alpha coefficients are 0.71, 0.91, $0.78,0.93,0.63$ and 0.92 for PWB, SFWB, EWB, FWB, BCS and FACT-B total respectively. Table 2 shows that FWB has highest Cronbach's coefficient, and BCS has

Table 2. Internal Consistency of the Iranian Version of FACT-B Scale $(\mathbf{N}=300)$

\begin{tabular}{lc}
\hline \multicolumn{2}{c}{ Cronbach's coefficient } \\
\hline PWB & 0.71 \\
SFWB & 0.91 \\
EWB & 0.78 \\
FWB & 0.93 \\
BCS & 0.63 \\
FACT-G & 0.92 \\
FACT-B & 0.92 \\
\hline
\end{tabular}

*PWB $=$ Physical Well-Being; FWB = Functional Well-Being; SFWB = Social and Family Well-Being; EWB =Emotional Well-Being; BCS=Breast Cancer Scale

Table 3. Internal Consistency of the Iranian Version of FACT-B Scale $(\mathbf{N}=300)$

\begin{tabular}{llllll}
\hline & PWB & EWB & SFWB & FWB & BCS \\
\hline PWB & 1 & & & & \\
EWB & $0.34 * *$ & 1 & & & \\
SFWB & 0.08 & $0.55^{* *}$ & 1 & & \\
FWB & $0.28^{* *}$ & $0.63 * *$ & $0.73^{* *}$ & 1 & \\
BCS & $0.47 * *$ & $0.61 * *$ & $0.37 * *$ & $0.52 * *$ & 1 \\
\hline
\end{tabular}

*PWB = Physical Well-Being; FWB = Functional Well-Being; SFWB = Social and Family Well-Being; EWB =Emotional Well-Being; BCS $=$ Breast Cancer Scale. ${ }^{* *} \mathrm{p}<0.01$

Table 4. Correlation Coefficients between Persian Version of FACT-B Factors with HADS Anxiety and Depression Scores $(\mathbf{N}=\mathbf{3 0 0})$

\begin{tabular}{lccccc}
\hline & PWB & EWB & SFWB & FWB & BCS \\
\hline Anxiety & $-0.52^{* *}$ & $-0.54^{* *}$ & $-0.30^{*}$ & $-0.34^{* *}$ & $-0.41^{* *}$ \\
depression & $-0.37^{* *}$ & $-.047^{* *}$ & $-0.36^{* *}$ & $-0.46^{* *}$ & $-0.44^{* *}$ \\
\hline
\end{tabular}

$*$ PWB $=$ Physical Well-Being; FWB = Functional Well-Being; SFWB = Social and Family Well-Being; EWB =Emotional Well-Being; BCS $=$ Breast Cancer Scale. ${ }^{* *} \mathrm{p}<0.01,{ }^{*} \mathrm{p}<0.05$

Table 6. Fitness indexes of Persian FACT-B

\begin{tabular}{cccccccl}
\hline NFI & CFI & GFI & AGFI & IFI & RMSEA & $X^{2} /$ df & $X^{2}$ \\
\hline 0.77 & 0.84 & 0.76 & 0.73 & 0.84 & 0.08 & 2.74 & 1586.35 \\
\hline
\end{tabular}

Validity

Interscale correlations: Convergent validity of the five subscales is assessed by examining the interscale correlations and Pearson's correlation with symptoms of anxiety and depression as assessed with the HADS

Interscale correlations between subscales of FACT-B shown at table 3. Except relationship between SFWB and $\mathrm{PWB}$, other subscales have significant correlation together.

\section{Discriminant validity}

Correlation coefficients between Persian version of FACT-B factors with HADS anxiety and depression are shown in table 4 . All of correlations of FACT-B subscales with anxiety and depression are negative and significant that this scale has discrimination validity (Table 4).

\section{Concurrent and construct validity}

For concurrent validity, correlation between FACT-B subscales and functional subscales of EROTC QLQ30 are calculated that shown in table 5. According to this table, PWB is significant correlation with Physical and other EROTC subscales; EWB is correlated to Emotion function and others of EROTC subscales; FWB is correlated to Physical function; BCS has significant correlation with all of EROTC subscales except cognition function, and FWB has no significant correlation with EROTC subscales.

Table 6 indicates the most important indices. Indicators of model fitness used include normed fit index (NFI=0.77), the comparative fit index $(\mathrm{CFI}=0.84)$, the goodness of fit index $(\mathrm{GFI}=0.76)$, the adjusted goodness of fit index (AGFI=0.73), incremental fit index $(\mathrm{IFI}=0.84)$, and the root-mean-square error of approximation (RMSEA=0.08). Chi 2 ratio on freedom degree is 2.74 that less than 3. Overall these findings indicate that the model fit is relatively modest.

\section{Discussion}

Given the importance of health-related quality of life in the lives of cancer patients, this study aims to investigate the factor structure and psychometric properties of the FACT-B scale. The findings of the study show that the reliability of Persian version is appropriate. Cronbach's alpha reliability coefficient for this scale, 0.71 for the physical well-being, social / family 0.91 , emotional 0.78 , functional 0.93 , breast subscale 0.63 and also total scale is 0.92 . The study of Akin et al (2008), shows that coefficients from the 0.57 to 0.77 . In the Korean version,

Table 5. Correlation Coefficients between Persian Version of FACT-B factors with EROTC QLQ30 (N =300)

\begin{tabular}{lllllc}
\hline & $\begin{array}{l}\text { Physical } \\
\text { function }\end{array}$ & $\begin{array}{c}\text { Role } \\
\text { function }\end{array}$ & $\begin{array}{l}\text { Emotion } \\
\text { function }\end{array}$ & $\begin{array}{c}\text { Cognition } \\
\text { function }\end{array}$ & $\begin{array}{c}\text { Social } \\
\text { function }\end{array}$ \\
\hline PWB & $0.416 * *$ & $0.514 * *$ & $0.576 * *$ & $0.275 * *$ & $0.621 * *$ \\
EWB & $0.445 * *$ & $0.297 * *$ & $0.460 * *$ & $0.408 * *$ & $0.447 * *$ \\
SFWB & 0.206 & 0.118 & 0.061 & 0.145 & 0.056 \\
FWB & $0.272 * *$ & 0.082 & 0.066 & 0.192 & 0.141 \\
BCS & $0.412 * *$ & $0.381 * *$ & $0.573 * *$ & 0.181 & 0.051 \\
\hline
\end{tabular}

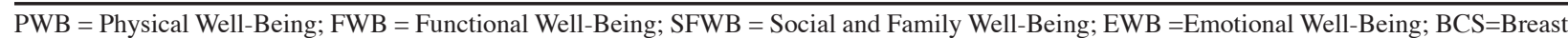
Cancer Scale. $* * \mathrm{p}<0.01$ 
the coefficients were from 0.67 for breast subscale to 0.86 for Social / Family (Yoo et al., 2005). In the original version as well as the coefficients for the subscales of breast 0.63 to 0.86 for the well-being subscale (Brady et al, 1997). In Wan study (2007) on the Chinese version of this scale the alpha coefficients for the breast subscale was 0.59. In the Malayalam version of this subscale, the breast subscale is lowest internal consistency coefficient (Pandey et al., 2002). Yoo (2005) to explain this finding suggested that the Indian community in issues related to relationships and sexuality are considered as taboo. Breast subscale Just a question about sex, but if the subject does not respond to a question from a small-scale retail. Also, Yoo et al (2005), in explaining the example of Korean breast cancer patients suggest that many patients may study them, have not resumed sexual activity after surgery and therefore comment on this aspect of the question for them is difficult. In Iranian culture, along with Yoo (2005) about Indian culture, discussions of sexuality are considered taboo, so due to this issue, as well as the average age of the participants, the lower the coefficient of this subscale seems justified

The other finding related to internal correlations between the subscales of the FACT-B. The results show that all correlations except correlation between family/social and physical well-being is significant. The overall correlation is moderate. For discrimination scale validity FACT-B, the relationship FACT-B subscales with depression and anxiety subscales were calculated. Findings indicate that all subscales of FACT-B are negative significant related to depression and anxiety, which expresses the reliability of the FACT-B scale suitable discriminant.

To examine the convergent validity of the FACT-B, the relationship between its subscales and QLQ30 functional subscales are calculated. Except Family / Social well-being, the rest of the FACT-B subscales are significantly correlated with QLQ30 functional subscales. In Glangkarn (2011), there is found significant relationship between the subscales social/family and social function subscale of EORTC. EORTC social functioning includes two questions that the concepts of social and physical conditions are considered. In contrast, social / family FACT-G consists of 7 questions that focus on social support and emotional closeness. In addition, each pair of comparisons, not only in the number of items, but some items are different in meaning. For example, the EORTC physical functioning consists of 5 items, however, only Q 4 (Staying in a bed or chair) and Question 7 on the scale of FACT-G (stay in bed) is similar. According to Smith (2007) perhaps the subscales of social / family well-being a measure of two factors related to the family, in particular the emotional support of family and family relationships and acceptance of the disease (items SFWB1 to SFWB5), and other factor is related to the primarily close personal relationships (SFWB6 and SFWB7). It seems the items of EORTC greatly focus on the physical domains, even at social functions and roles. On the other hand, functional well-being FACT-G, have a much wider range of activities, both work and rest and enjoy life covers the aspects which need not be related to physical function. Both questionnaires EORTC QLQ-BR23 and the FACT-B, that have additional questions specific to breast cancer. However, it seems FACT unlike to EORTC lack focuses on the adverse effects of systemic therapy, including breast symptoms, dry mouth, hot flashes, and headache (GlankKaren, 2011).

In conclusion, the results show that the FACT-B scale translation is a valid and reliable tool for measuring HRQoL among Iranian breast cancer patients. However, this study has limitations. It is recommended that this scale should be applied to determine the patients, quality of life during different stages. Also suggested that this scale should be used in breast cancer patients at different ages to be determined the sensitivity of the scale..

\section{References}

Aaronson NK et al (1993). The european organization for research and treatment of cancer QLQ-C30: a quality-of-life instrument for use in international clinical trials in oncology. $J$ National Cancer Institute, 85, 365-76.

Akin S, Can G , Durna, Z, Aydiner A (2008). The quality of life and self-efficacy of Turkish breast cancer patients undergoing chemotherapy. Eur J Oncol Nurs, 12, 449-56.

Brady MJ, Cella DF et al (1997). Reliability and validity of the Functional Assessment of Cancer Therapy-Breast qualityof-life instrument. J Clin Oncol, 15, 974-86.

Cella DF, Tulsky DS (1993). Quality of life in cancer: Definition, purpose, and method of measurement. Cancer Investigation, 11, 327-36.

Cella DF (1997). FACIT manual: Manual of the Functional Assessment of Chronic Illness Therapy (FACIT) measurement system. CORE, Evanston.

Cella DF, Tulsky DS, Gray G, et al (1993). The Functional Assessment of Cancer Therapy scale: development and validation of the general measure. J Clin Oncol, 11, 570-9.

Fayers P, Aaronson N, Bjordal K, et al (2001). EORTC QLQ-C30 scoring manual, EORTC Quality of Life Group 3rd edn. EORTC, Brussels, Belgium.

Glangkarn S, Promasatayaprot V, Porock D, Edgley A (2011). Measuring quality of life in Thai women with breast cancer. Asian Pac J Cancer Prev, 12, 637-44

Pandey M, Thomas BC, Ramdas K, Eremenco S, Nair MK (2002). Quality of life in breast cancer patients: Validation of a FACT-B Malayalam version. Qual Life Res, 11, 87-90.

Manandhar S, Shrestha DS, Taechaboonsermsk P, Siri S, Suparp J (2014). Quality of life among breast cancer patients undergoing treatment in national cancer centers in Nepal. Asian Pac J Cancer Prev, 15, 9753-7.

Montazeri A, Harirchi I, Vahdani M, et al (1999). The european organization for research and treatment of cancer quality of life questionnaire (EORTC QLQ-C30): translation and validation study of the Iranian version. Support Care Cancer, 7, 400-6.

Montazeri A, Vahdaninia M, Ebrahimi M, Jarvandi S (2003). The Hospital anxiety and depression scale (HADS): translation and validation study of the Iranian version. Health and Quality of Life Outcomes, 1, 14.

Montazeri A (2008). Health-related quality of life in breast cancer patients: a bibliographic review of the literature from 1974 to 2007, J Experimental Clin Cancer Res, 27-32.

Munoz M (2010). Quality of life during treatment in young women with breast cancer. Breast Cancer Res Treat, 123, 75-7.

Ramadas A, Qureshi AM, Dominic NA, et al (2015). Socio- 
demography and medical history as predictors of healthrelated quality of life of breast cancer survivors. Asian Pac $J$ Cancer Prev, 6, 1479-85.

Smith AB, Wright P, Selby PJ, Velikova G (2007) .A Rasch and factor analysis of the Functional Assessment of Cancer Therapy-General (FACT-G). Health and Quality of Life Outcomes, 5, 19.

Victorson D, Barocas J, Song J, Cella D (2008) .Reliability across studies from the functional assessment of cancer therapy-general (FACT-G) and its subscales: a reliability generalization. Qual Life Res, 17, 1137-46.

Wan C. (2007). Validation of the simplified Chinese version of the FACT-B for measuring quality of life for patients with breast cancer. Breast Cancer Res Treat, 106, 413-8.

Yanez B, Pearman T, Lis CG, Beaumont JL, Cella D (2013). The FACT-G7: a rapid version of the functional assessment of cancer therapy-general (FACT-G) for monitoring symptoms and concerns in oncology practice and research. Ann Oncol, 24, 1073-8.

Yoo HJ, Ahn SH, Eremenco S, et al (2005). Korean translation and validation of the functional assessment of cancer therapy-breast (FACT-B) scale version 4. Quality of Life Research, 14, 1627-32. 